\title{
Quantification of ascorbic acid and sodium ascorbate in powder blends for tableting and in vitamin $C$ chewable tablets by NIR-chemometry
}

\author{
IOAN TOMUȚĂ ${ }^{1 *}$ \\ DANA DUDAŞ ${ }^{1}$ \\ ANDREEA LOREDANA VONICA ${ }^{2}$ \\ SORIN E. LEUCUŢA ${ }^{1}$ \\ ${ }^{1}$ Iuliu Hatieganu University of Medicine \\ and Pharmacy, Department of \\ Pharmaceutical Technology and \\ Biopharmaceutics, 400023 Cluj-Napoca \\ Romania \\ 2 S.C. Polipharma Industries, 550052 \\ Sibiu, Romania
}

Accepted June 4, 2013

\begin{abstract}
The paper proposes a near infrared method able to directly and simultaneously quantify ascorbic acid and sodium ascorbate in powder blends for tableting and in vitamin $\mathrm{C}$ chewable tablets without any sample preparation. In the first step, calibration models for the quantification of ascorbic acid and sodium ascorbate in powder blends for tableting and subsequently in chewable vitamin $\mathrm{C}$ tablets (corresponding to $80-120 \%$ active substance) were developed according to an experimental design with 2 variables and 5 levels. Then, using the best calibration models, the methods were fully validated in terms of recovery, precision and accuracy for both powder blends and vitamin $C$ chewable tablets. The validated concentration range was $15.14-18.51 \%$ for ascorbic acid and $12.06-14.49 \%$ for sodium ascorbate in powder blends and 91.85-111.03 mg per tablet for ascorbic acid and 71.01-84.50 mg per tablet for sodium ascorbate in tablets. Validation results showed good precision and accuracy.
\end{abstract}

Keywords: NIR spectroscopy, chemometrics, ascorbic acid, sodium ascorbate, validation, powder blends

Pharmaceutical controls are time-consuming and require extensive sample preparation. Process analytical technology (PAT) implementation in the pharmaceutical industry would reduce these time consuming operations. To perform this implementation, the use of analytical techniques capable of providing accurate results in a simple and rapid manner is necessary (1). Other advantages include little or no sample preparation $(2,3)$. Near infrared spectrophotometry (NIR) is a non-destructive and non-invasive technique that allows determination of chemical properties such as active pharmaceutical ingredient (API) or excipient content.

\footnotetext{
* Correspondence; e-mail: tomutaioan@umfcluj.ro
} 
NIR spectroscopy can be used to perform quantitative determinations of one, two or more compounds in complex matrices like, for instance, a pharmaceutical powder blend for tableting or tablets. A NIR assay method is usually not developed in the »traditional« way as UV spectrometry or HPLC assay methods (4). A major step to develop a NIR assay method is the calibration procedure for model development. Once calibration is developed and favourable predictions are expected, they must be validated to be accepted for routine use. For external validation, independent sets of samples are needed. There are several validation parameters that must be determined in order to be consistent with the recommendations of official guidelines: accuracy, precision (repeatability and intermediate precision), specificity, linearity and range of application $(5,6)$.

Many papers report the determination of API content in powder blends or tablets by NIR methods $(6,7)$, but only a few are focused on the prediction of two or more APIs and/or excipients combined in powder blends or tablets $(8,9)$.

The methods currently used to determine the amount of ascorbic acid and sodium ascorbate in tablets or powder blends for tableting are $\operatorname{HPLC}(10,11)$ and titrimetry $(12$, $13)$, but they have the disadvantage of being rather time-consuming. Unlike the conventional methods, NIR-chemometric methods are faster and do not require prior preparation of the sample, so they can be a viable alternative for direct quantification of ascorbic acid and sodium ascorbate in powder blends for tableting and vitamin $C$ tablets. The first objective of our research was to develop a NIR-chemometric method suitable for direct quantification of both APIs from powder blends for tableting.

\section{EXPERIMENTAL}

\section{Materials}

Ascorbic acid and sodium ascorbate (NPCP, China), sorbitol and mannitol (Roquette, France), orange flavour (Esarom, Romania), sodium saccharin (Niutang Chemical, China), sodium cyclamate (Golden Time Enterprise, China), silicon dioxide - Aerosil (RohmPharma Polymers, Germany) and magnesium stearate (Mosselman, Belgium) were used.

\section{Sample preparation}

For calibration purposes, powder blends for vitamin $\mathrm{C}$ chewable tablets were prepared. Ascorbic acid, sodium ascorbate, sorbitol, mannitol, sodium saccharin, sodium cyclamate, orange flavour and silicon dioxide were mixed for 5 minutes using a planetary mixer (PRS type, Erweka, Germany) and then passed through a 0.800-mm sieve. Magnesium stearate was then added and mixing was continued for one more minute. The mixture composition was designed for a tablet of a total mass of approximately 600 $\mathrm{mg}$ and for $180 \mathrm{mg}$ vitamin C (consisting of $50 \%$ ascorbic acid and $50 \%$ sodium ascorbate in the tablet). This formulation will be further considered as the $100 \%$ active content formulation. Quantitative and qualitative composition of vitamin C chewable tablets are presented in Table I. 
I. Tomuţă et al:: Quantification of ascorbic acid and sodium ascorbate in powder blends for tableting and in vitamin C chewable tablets by NIR-chemometry, Acta Pharm. 63 (2013) 373-384.

Table I. Composition of calibration and validation samples

\begin{tabular}{lcccrr}
\hline Content (\%) & $80^{\mathrm{a}}$ & $90^{\mathrm{a}, \mathrm{b}}$ & $100^{\mathrm{a}, \mathrm{b}}$ & $110^{\mathrm{a}, \mathrm{b}}$ & $120^{\mathrm{a}}$ \\
\hline Ascorbic Acid $97 \%$ & Vitamin C chewable & tablet composition $(m g$ per & tablet $)$ & \\
Sodium ascorbate & 82.4 & 92.7 & 103.0 & 113.3 & 123.6 \\
Sorbitol & 72.0 & 81.0 & 90.0 & 99.0 & 108.0 \\
Mannitol & 341.8 & 322.5 & 303.2 & 283.9 & 264.6 \\
Orange flavour & 90.0 & 90.0 & 90.0 & 90.0 & 90.0 \\
Sodium saccharin & 1.5 & 1.5 & 1.5 & 1.5 & 1.5 \\
Sodium cyclamate & 0.3 & 0.3 & 0.3 & 0.3 & 0.3 \\
Silicon dioxide & 3.0 & 3.0 & 3.0 & 3.0 & 3.0 \\
Magnesium stearate & 3.0 & 3.0 & 3.0 & 3.0 & 3.0 \\
\hline Tablet mass (mg) & 6.0 & 6.0 & 6.0 & 6.0 & 6.0 \\
\hline
\end{tabular}

a Calibration samples: levels 1-2-3-4-5.

b Validation samples: levels 2-3-4.

\section{Calibration and validation protocol}

The calibration set used included 28 different formulations corresponding to 80,90 , 100, 110 and $120 \%$ ascorbic acid and sodium ascorbate. The samples were prepared according to an experimental design with two factors and 5 levels (Table II) on different days, in the run order. This protocol included batches and days as sources of variability for calibration steps.

To validate the NIR methods for the assay of ascorbic acid and sodium ascorbate, the same formulations as for the calibration set, corresponding to 90,100 and $110 \%$ ascorbic acid and sodium ascorbate (Table II, samples N7, N13 and N19), were manufactured. At each of the three concentration levels four replicate samples were prepared. In order to include batches and days as sources of variability in validation steps, this protocol was repeated three times on three different days, resulting in a total of 36 validation samples.

\section{NIR spectra recording}

Powder blends for tableting were analyzed in a reflection mode using a FT-NIR analyzer (Antaris II, ThermoElectronScientific, USA) equipped with a sample cup spinner for inhomogenous samples, an integrating sphere and an indium gallium arsenide (InGaAs) detector. Reflection spectra were collected with OMNIC software (Thermo Scientific, USA). Each spectrum was the average of 32 scans and the resolution was $8 \mathrm{~cm}^{-1}$ over a range from 11000 to $4000 \mathrm{~cm}^{-1}$. Each powder sample was analyzed three times.

From each sample, fifteen different intact tablets were analyzed in reflection mode using the same FT-NIR analyzer equipped with a universal tablet holder. 
I. Tomuţă et al.: Quantification of ascorbic acid and sodium ascorbate in powder blends for tableting and in vitamin C chewable tablets by NIR-chemometry, Acta Pharm. 63 (2013) 373-384.

Table II. Composition of the calibration set, according to a $2^{5}$ experimental design

\begin{tabular}{crrrrr}
\hline Code & X1 & X2 & Code & X1 & X2 \\
\hline N1 & 82.4 & 72.0 & N15 & 123.6 & 90.0 \\
N2 & 92.7 & 72.0 & N16 & 82.4 & 99.0 \\
N3 & 103.0 & 72.0 & N17 & 92.7 & 99.0 \\
N4 & 113.3 & 72.0 & N18 & 103.0 & 99.0 \\
N5 & 123.6 & 72.0 & N19c & 113.3 & 99.0 \\
N6 & 82.4 & 81.0 & N20 & 123.6 & 99.0 \\
N7 & 92.7 & 81.0 & $\mathrm{~N} 21$ & 82.4 & 108.0 \\
N8 & 103.0 & 81.0 & N22 & 92.7 & 108.0 \\
N9 & 113.3 & 81.0 & $\mathrm{~N} 23$ & 103.8 & 108.0 \\
N10 & 123.6 & 81.0 & $\mathrm{~N} 24$ & 113.3 & 108.0 \\
N11 & 82.4 & 90.0 & $\mathrm{~N} 25$ & 123.6 & 108.0 \\
N12 & 92.7 & 90.0 & $\mathrm{~N} 26$ & 103.0 & 90.0 \\
N13 & 103.0 & 90.0 & $\mathrm{~N} 27$ & 103.0 & 90.0 \\
N14 & 113.3 & 90.0 & $\mathrm{~N} 28$ & 103.0 & 90.0 \\
\hline
\end{tabular}

$\mathrm{X} 1$ - amount of ascorbic acid (mg per tablet); X2 - amount of sodium ascorbate (mg per tablet)

a Level 1 of validation samples (X1 = 90\%; X2 = 90\%);

b Level 2 of validation samples (X1 = 100\%; X2 = 100\%);

${ }^{c}$ Level 3 of validation samples (X1 = 110\%; X2 = $\left.110 \%\right)$.

\section{Spectral data analysis}

Development of NIR calibration models is an iterative process and consists of checking different spectral pretreatments, as well as their combinations within different spectral ranges. Both the specific spectral regions containing strong bands or the whole spectral range and different spectral pretreatments were tested in order to develop calibration models for ascorbic acid and sodium ascorbate assay in powder blends for tableting and tablets. Development of models was based on the following spectral pretreatments: constant offset elimination (COE), straight line subtraction (SLS), standard normal variate (SNV), minim-maxim normalization $(\mathrm{MMN})$, multiplicative scatter correction (MSC), first-derivative (FD), second-derivative (SD) or combined methods: FD followed by SLS, FD followed by SNV, FD followed by MSC.

The QUANT package of the OPUS 6.5 software package was used for multivariate regressions, with no spectra pretreatment and after applying pre-processing methods. This software allows model validation by full cross-validation and test-validation. For the development of prediction models, the PLS (partial least squares) regression method was used. The models for ascorbic acid and sodium ascorbate assay in powder blends were validated by the full cross-validation method while the models for ascorbic acid and sodium ascorbate assay in tablets were validated by the test-validation method. The model with the highest predictive potential was selected according to conventional criteria: low root mean square error of cross validation (RMSECV), low root mean square er- 
I. Tomuţă et al: Quantification of ascorbic acid and sodium ascorbate in powder blends for tableting and in vitamin C chewable tablets by NIR-chemometry, Acta Pharm. 63 (2013) 373-384.

ror of estimation (RMSEE), high coefficient of determination $\left(R^{2}\right)$, small number of PLS factors, and low bias (14).

\section{RESULTS AND DISCUSSION}

\section{Ascorbic acid and sodium ascorbate assay in powder blends for tableting: development of models and validation}

Twenty eight powder blends containing both ascorbic acid and sodium ascorbate and a NIR-chemometric method to simultaneously and directly quantify the two components was developed and validated.

Model development for API assay consisted of checking different combinations of spectrum pretreatments in association with different selected spectrum regions. PLS regression was performed with the calibration set and cross-validation was carried out for model validation. The first step in model development was to investigate eleven pre-processing methods [models (a)-(k), Table III]. This investigation and the selection of the model with the highest predictive potential were mainly based upon the choice of the number of factors (main components), and calculation of the root mean square error of cross validation (RMSECV), root mean square error of estimation (RMSEE), bias and $R^{2}$ (15). The models studied for ascorbic acid and sodium ascorbate assay in powder blends and their most important characteristics are given in Table III.

The best results obtained after examining different spectral regions were for the models created using the spectral region $10000-4133 \mathrm{~cm}^{-1}$ for ascorbic acid and 10000-4480 $\mathrm{cm}^{-1}$ for sodium ascorbate. Among the models presented in Table III, it can be seen that for ascorbic acid, model (j) has better prediction ability than other models. The (j) model has higher values for $R^{2}$ and lower values for RMSECV and bias than other models, notably models $(\mathrm{g}),(\mathrm{h})$ and $(\mathrm{k})$. At the same time, it can be seen also in Table III, that for sodium ascorbate, model $(\mathrm{h})$ is the model with a better prediction ability. Based on the mentioned predictive ability criteria (low RMSEC, high $R^{2}$, low number of PLS factors and low bias), model (j) was selected as the best fitting model for the ascorbic acid assay and model (h) was selected as the best fitting model for the sodium ascorbate assay.

The predictive ability of the chosen models was checked on independent samples during the method validation step. Accuracy, precision (repeatability and intermediate precision), linearity and specific range of application were the validation parameters. Validation was performed according to the strategy proposed by Hubert et al. $(16,17)$. This approach uses tolerance intervals as statistical methodology, which allows predicting a region of concentrations where each future result has a probability to be defined by the analyst (18). For validation purposes, independent batches comprising 4 replicates of powder blends for tabletting at 3 different active levels of APIs (90, 100, and $110 \%, \mathrm{~m} / \mathrm{m}$, formulations N7, N13, N23) were prepared and analyzed on 3 different days, resulting in a total of 36 samples. The accuracy profile has the advantage of taking into account the total error, which is the sum of trueness (systematic error) and precision (random error) $(16,17)$. 
I. Tomuţă et al.: Quantification of ascorbic acid and sodium ascorbate in powder blends for tableting and in vitamin C chewable tablets by NIR-chemometry, Acta Pharm. 63 (2013) 373-384.

Table III. Type of spectral pre-treatment, $\mathrm{R}^{2}$, spectral range selected, number of PLS factors and RMSECV of different models for ascorbic acid and sodium ascorbate assays in powder blends for tableting

\begin{tabular}{|c|c|c|c|c|c|c|c|c|c|c|c|}
\hline Model & (a) & (b) & (c) & (d) & (e) & (f) & (g) & (h) & (i) & (j) & $(\mathrm{k})$ \\
\hline Pre-treatment & None & COE & SLS & SNV & MMN & MSC & FD & SD & $\begin{array}{l}\text { FS+ } \\
+ \text { SLS }\end{array}$ & $\begin{array}{l}\text { FD+ } \\
+ \text { SVN }\end{array}$ & $\begin{array}{c}\text { FD+ } \\
+\mathrm{MSC}\end{array}$ \\
\hline \multicolumn{12}{|c|}{ Ascorbic acid } \\
\hline \multicolumn{2}{|c|}{ Spectral range selected $\left(\mathrm{cm}^{-1}\right)$} & \multicolumn{10}{|c|}{$10000-4133$} \\
\hline Number of PLS factors & 10 & 10 & 9 & 9 & 9 & 9 & 10 & 8 & 10 & 10 & 10 \\
\hline$R^{2}$ & 90.58 & 91.38 & 92.61 & 93.97 & 92.62 & 93.92 & 94.33 & 95.98 & 94.61 & 96.75 & 95.49 \\
\hline RMSECV & 0.687 & 0.664 & 0.612 & 0.559 & 0.633 & 0.561 & 0.540 & 0.452 & 0.525 & 0.396 & 0.478 \\
\hline Bias $(\%)$ & 0.011 & 0.008 & 0.016 & 0.010 & 0.019 & 0.013 & 0.004 & 0.007 & 0.004 & 0.004 & 0.016 \\
\hline \multicolumn{12}{|c|}{ Sodium ascorbate } \\
\hline \multicolumn{6}{|c|}{ Spectral range selected $\left(\mathrm{cm}^{-1}\right)$} & \multicolumn{3}{|c|}{$10000-4480$} & & & \\
\hline Number of PLS factors & 10 & 9 & 10 & 10 & 9 & 10 & 10 & 10 & 10 & 10 & 10 \\
\hline$R^{2}$ & 91.29 & 91.7 & 94.18 & 94.62 & 94.69 & 94.21 & 97.13 & 98.19 & 97.65 & 97.81 & 97.34 \\
\hline RMSECV & 0.528 & 0.516 & 0.428 & 0.408 & 0.407 & 0.427 & 0.300 & 0.242 & 0.268 & 0.262 & 0.290 \\
\hline Bias (\%) & 0.017 & 0.007 & 0.003 & 0.005 & 0.021 & 0.004 & 0.018 & 0.009 & 0.017 & 0.017 & 0.014 \\
\hline
\end{tabular}

No spectral pre-treatment.

COE - constant offset elimination, SLS - straight line subtraction, SNV - standard normal variate, MMN - minim-maxim normalization, MSC - multiplicative scatter correction, FD - first derivative, SD - second derivative, FS+SLS-first derivative followed by straight line subtraction, FD+SVN - first derivative followed by standard normal variate, FD+MSC - first derivative followed by multiplicative scatter correction.

The validation criteria of the methods developed for ascorbic acid and sodium ascorbate quantitative analysis in powder blends for tableting are given in Table IV. The trueness of the method was evaluated by calculating recovery. The values obtained for recovery were satisfactory for both APIs at all three concentration levels tested, all values being included in the range of 98.8 to $101.7 \%$ (Table IV). The precision of the methods was evaluated by calculating repeatability at the three concentration levels (Table IV). For ascorbic acid, the RSD repeatability ranged from 1.5 to $2.0 \%$, and for sodium ascorbate, it was $1.2-1.9 \%$.

Fig. 1a shows the accuracy profile for the ascorbic acid assay in powder blends for tableting, which is based on the validation results obtained with the developed NIR (j) model, FD followed by SNV spectra pretreatments, in the spectral region 10000-4133 $\mathrm{cm}^{-1}$. Similarly, the accuracy profile for the sodium ascorbate assay in powder blends for tableting was built using validation results obtained with the developed NIR (h) model, SD spectra pretreatments, in the spectral region 10000-4480 $\mathrm{cm}^{-1}$ (Fig. 1b). As shown in Fig. 1a and in Table IV, accuracy of the method for ascorbic acid in powder blends is good, as the $\beta$-expectation tolerance limits do not exceed the $\pm 5 \%$ acceptance limits. The largest relative tolerance limits were $\pm 5.0 \%$, for the lowest ascorbic acid content of pow- 
I. Tomuţă et al.: Quantification of ascorbic acid and sodium ascorbate in powder blends for tableting and in vitamin C chewable tablets by NIR-chemometry, Acta Pharm. 63 (2013) 373-384.

Table IV. Validation results of NIR methods for ascorbic acid and sodium ascorbate assays in powder blends for tableting

\begin{tabular}{ccccc}
\hline \multirow{2}{*}{$\begin{array}{c}\text { Mean API } \\
(\%)\end{array}$} & $\begin{array}{c}\text { Recovery } \\
(\%)\end{array}$ & $\begin{array}{c}\text { Repeatability } \\
(\text { RSD }, \%)\end{array}$ & $\begin{array}{c}\text { Relative tolerance } \\
\text { limits }(\%)\end{array}$ & $\begin{array}{c}\text { Tolerance } \\
\text { limits }(\%)\end{array}$ \\
\hline \multicolumn{5}{c}{} \\
& Ascorbic acid & \\
\hline 15.14 & 100.0 & 2.0 & {$[-5.0,5.0]$} & {$[14.4,15.9]$} \\
16.83 & 99.5 & 1.5 & {$[-4.7,3.8]$} & {$[16.0,17.5]$} \\
18.51 & 101.7 & 1.6 & {$[-3.3,4.7]$} & {$[17.9,19.4]$} \\
\hline \multicolumn{5}{c}{ Sodium ascorbate } \\
\hline 12.06 & 100.5 & 1.9 & {$[-4.6,5.6]$} & {$[11.5,12.7]$} \\
13.30 & 99.7 & 1.7 & {$[-5.0,4.4]$} & {$[12.7,13.9]$} \\
14.49 & 98.8 & 1.2 & {$[-5.0,2.6]$} & {$[13.9,15.0]$} \\
\hline
\end{tabular}
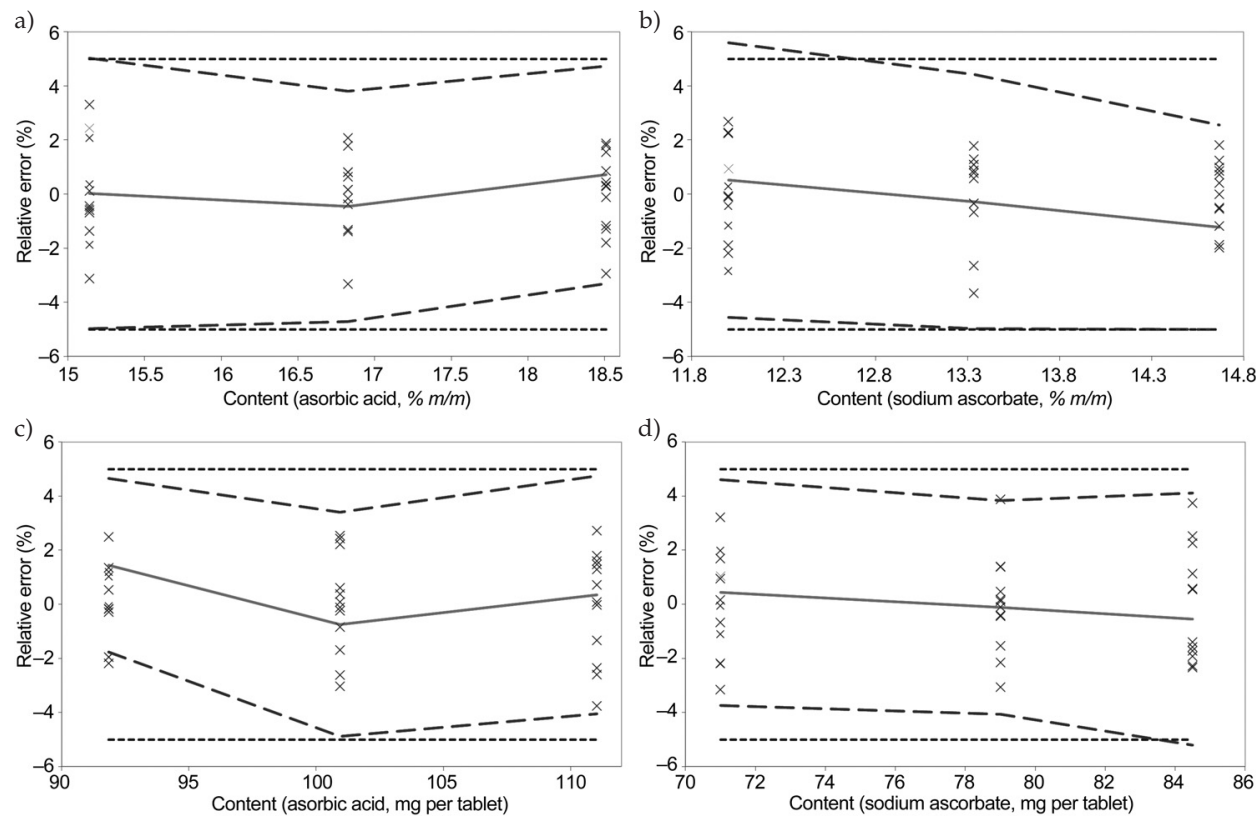

Fig. 1. Accuracy profile of the NIR methods for: a) ascorbic acid, b) sodium ascorbate assay in powder blend for tableting, c) ascorbic acid and d) sodium ascorbate assay in tablets. The full line is relative bias, dashed lines are $\beta$-expectation tolerance limits $(\beta=95 \%)$ and dotted curves are acceptance limits $( \pm 5 \%)$. 
der blends in validation batches. Similarly, the accuracy of the method for sodium ascorbate in powder blends seems quite good; the $\beta$-expectation tolerance limits exceeded only slightly the $\pm 5 \%$ acceptance limits (Fig. $1 \mathrm{~b}$ and Table IV). The largest relative tolerance limits were $-5.0 \%$ for the highest sodium ascorbate content and $5.6 \%$, for the lowest sodium ascorbate content of powder blends in validation batches. Accuracy profiles included in the $\pm 5 \%$ limits give enough confidence for the quality and reliability of the results obtained by the developed NIR-chemometric methods (19).

The linear profile of the prediction models is shown in Figs. 2a and b. The linear model was obtained by plotting calculated concentrations of the validation samples as a function of introduced concentrations. Dashed limits correspond to the accuracy profile and dotted curves represent the acceptance limits at $\pm 5 \%$ expressed in concentration units. The linearity profile is within or close to the acceptance limits, the $R^{2}$ value is higher than 0.95 and the slope is very close to 1 , confirming the linearity of the models for ascorbic acid and sodium ascorbate assays in powder blends for tableting.

a)

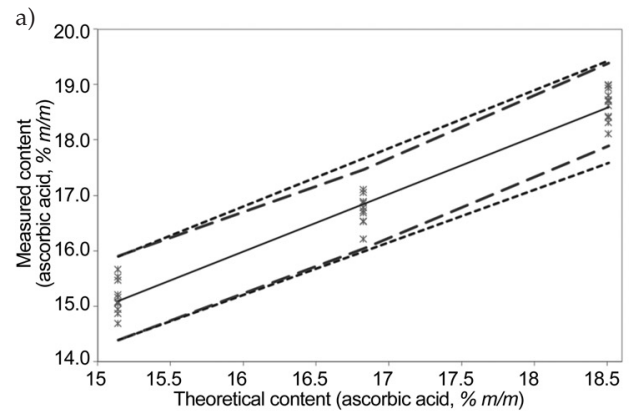

c)

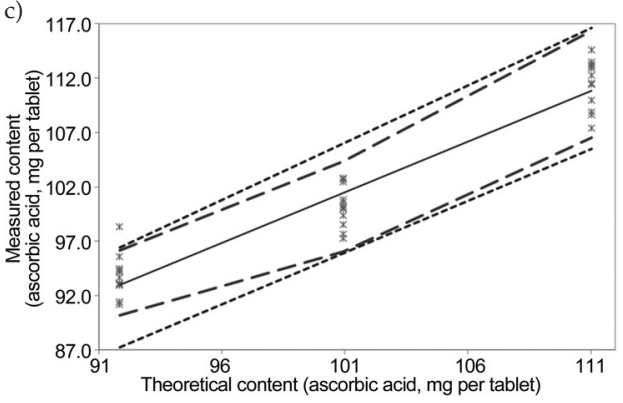

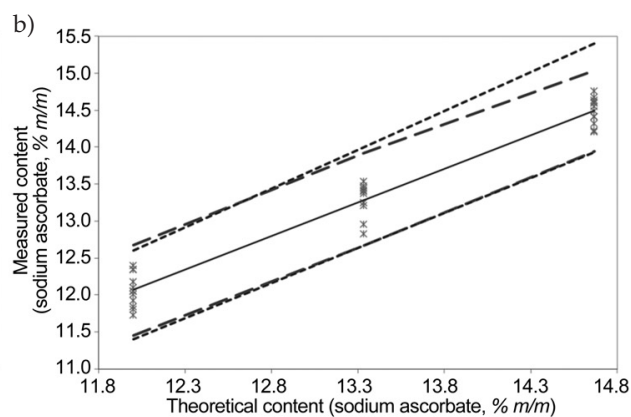

d)

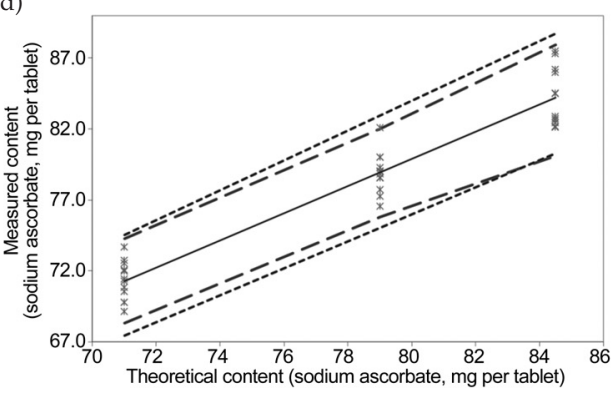

Fig. 2. Linear profile of the NIR methods for: a) ascorbic acid, b) sodium ascorbate assay in powder blend for tableting, c) ascorbic acid and d) sodium ascorbate assays in tablets. Dashed limits correspond to the accuracy profile and dotted curves represent acceptance limits at $\pm 5 \%$. Full line is the identity line, $\mathrm{y}=\mathrm{x}$. 
I. Tomuţă et al.: Quantification of ascorbic acid and sodium ascorbate in powder blends for tableting and in vitamin C chewable tablets by NIR-chemometry, Acta Pharm. 63 (2013) 373-384.

\section{Ascorbic acid and sodium ascorbate assay in vitamin $C$ chewable tablets: development of models and validation}

The other aim of our research was to develop a NIR-chemometric method suitable for direct quantification of ascorbic acid and sodium ascorbate in tablets. For this purpose, a protocol consisting of preparing and analyzing 28 of tablet formulations was followed. Practically, the powder blends used for the development and validation of NIR chemometric methods in powder blends for tableting were compressed into vitamin C chewable tablets with a total mass of $600 \mathrm{mg}$ per tablet. The same protocol and methodology as previously described for the development and validation of NIR chemometric methods to simultaneously and directly quantify ascorbic acid and sodium ascorbate in powder blends for tableting was followed for tablets.

Table $\mathrm{V}$ shows the main characteristics of the investigated models for ascorbic acid and sodium ascorbate assays in tablets.

Based on the analysis of different spectral regions, the models generated with the use of spectral region $8850-4070 \mathrm{~cm}^{-1}$ for both ascorbic acid and sodium ascorbate showed the best results. It can be seen from Table V that for ascorbic acid (j) model is better than other models in terms of prediction. The (j) model has higher $R^{2}$ values and lower

Table V. Type of spectral pre-treatment, $\mathrm{R}^{2}$, spectral range selected, number of PLS factors, RMSEP of different models for ascorbic acid and sodium ascorbate assays in tablets

\begin{tabular}{|c|c|c|c|c|c|c|c|c|c|c|c|}
\hline Model & (a) & (b) & (c) & (d) & (e) & (f) & (g) & (h) & (i) & (j) & $(\mathrm{k})$ \\
\hline Pre-treatment & None & $\mathrm{COE}$ & SLS & SNV & MMN & MSC & FD & SD & $\begin{array}{c}\mathrm{FS}+ \\
+\mathrm{SLS}\end{array}$ & $\begin{array}{c}\text { FD+ } \\
+ \text { SVN }\end{array}$ & $\begin{array}{c}\text { FD+ } \\
+\mathrm{MSC}\end{array}$ \\
\hline \multicolumn{12}{|c|}{ Ascorbic acid } \\
\hline \multicolumn{2}{|c|}{ Spectral range selected $\left(\mathrm{cm}^{-1}\right)$} & \multicolumn{10}{|c|}{$8850-4070$} \\
\hline Number of PLS factors & 8 & 8 & 8 & 7 & 8 & 8 & 10 & 9 & 10 & 10 & 10 \\
\hline$R^{2}$ & 82.75 & 84.08 & 85.31 & 84.49 & 86.55 & 86.19 & 92.16 & 85.06 & 91.81 & 95.31 & 94.95 \\
\hline RMSEP & 7.10 & 6.92 & 6.76 & 6.87 & 6.72 & 6.64 & 5.74 & 6.79 & 5.80 & 5.21 & 5.28 \\
\hline Bias (\%) & -0.541 & -0.551 & -0.478 & -0.631 & -1.22 & -1.12 & -0.14 & 0.24 & -0.109 & -0.097 & -0.15 \\
\hline \multicolumn{12}{|c|}{ Sodium ascorbate } \\
\hline \multicolumn{6}{|c|}{ Spectral range selected $\left(\mathrm{cm}^{-1}\right)$} & \multicolumn{3}{|c|}{$8850-4070$} & & & \\
\hline Number of PLS factors & 9 & 10 & 10 & 9 & 9 & 9 & 9 & 8 & 9 & 8 & 8 \\
\hline$R^{2}$ & 87.50 & 95.37 & 95.49 & 93.71 & 93.85 & 93.68 & 95.69 & 93.50 & 96.18 & 95.14 & 94.87 \\
\hline RMSEP & 3.87 & 3.40 & 3.38 & 3.68 & 3.66 & 3.68 & 3.34 & 3.71 & 3.25 & 3.44 & 3.49 \\
\hline Bias (\%) & 0.127 & -2.68 & -0.201 & -0.255 & -0.409 & -0.27 & -0.36 & -0.46 & -0.262 & -0.327 & -0.386 \\
\hline
\end{tabular}

No spectral pre-treatment.

COE - constant offset elimination, SLS - straight line subtraction, SNV - standard normal variate, MMN - minim-maxim normalization, MSC - multiplicative scatter correction, FD - first derivative, SD - second derivative, FS+SLS - first derivative followed by straight line subtraction, FD+SVN - first derivative followed by standard normal variate, FD+MSC - first derivative followed by multiplicative scatter correction. 
I. Tomuţă et al.: Quantification of ascorbic acid and sodium ascorbate in powder blends for tableting and in vitamin C chewable tablets by NIR-chemometry, Acta Pharm. 63 (2013) 373-384.

values for RMSEP and bias than other models, especially (i) and (k) models. Regarding sodium ascorbate, the (i) models prediction ability is better than any one of other models presented in Table V. On these grounds, the best fitting model chosen for the ascorbic acid assay in tablets was model (j) with FD followed by SNV pre-treatment and model (i) with FD followed by SLS pre-treatment was chosen as the most suitable model for the sodium ascorbate assay in tablets.

Table VI shows validation criteria of the developed methods for ascorbic acid and sodium ascorbate assays in tablets. Recovery had satisfactory values at all three concentration levels for both APIs, the lowest value being $98.9 \%$ and the highest $101.5 \%$ (Table VI). The precision of the methods was evaluated by calculating repeatability at the three concentration levels used. Repeatability was good for all studied samples, parameters had satisfactory values at all concentration levels for both APIs, ascorbic acid and sodium ascorbate (Table VI). RSD ranged from 1.6-1.9 \% for ascorbic acid and 1.7-2.1\% for Na-ascorbate.

Accuracy profiles for ascorbic acid and sodium ascorbate assays in tablets are given in Figs. 1c and d, respectively. The accuracy profile for the ascorbic acid assay is based on the validation results obtained with the NIR (j) model, FD followed by SNV spectra pretreatments, in the spectral region $8850-4070 \mathrm{~cm}^{-1}$, while the accuracy profile for the sodium ascorbate assay is based on the validation results obtained with the NIR (i) model, with FD followed by SLS spectra pretreatments, in the same spectral region. Since $\beta$-expectation tolerance limits are maintained within $\pm 5 \%$ acceptance limits, the accuracy of the method for the ascorbic acid assay in tablets is considered good (Fig. 1c and Table VI. The largest relative tolerance limits were $-4.9 \%$ for the medium ascorbic acid content and $4.8 \%$ for the highest ascorbic acid content of tablets in validation batches. Similarly to the accuracy of the method for sodium ascorbate assay tablets is rather good, the $\pm 5 \%$ (19) acceptance limits being only slightly exceeded (Fig. 1d) and Table VI). The largest relative tolerance limits were $-5.2 \%$ for the highest sodium ascorbate

Table VI. Validation results of NIR methods for ascorbic acid and sodium ascorbate assays in tablets

\begin{tabular}{|c|c|c|c|c|}
\hline \multirow[b]{2}{*}{$\begin{array}{l}\text { Mean API content } \\
\text { (mg per tablet) }\end{array}$} & \multirow[b]{2}{*}{$\begin{array}{l}\text { Recovery } \\
(\%)\end{array}$} & \multirow[b]{2}{*}{$\begin{array}{l}\text { Repeatability } \\
\text { (RSD, \%) }\end{array}$} & \multicolumn{2}{|c|}{ Accuracy } \\
\hline & & & $\begin{array}{l}\text { Relative tolerance } \\
\text { limits }(\%)\end{array}$ & $\begin{array}{l}\text { Tolerance limits } \\
\text { (mg per tablet) }\end{array}$ \\
\hline \multicolumn{5}{|c|}{ Ascorbic acid } \\
\hline 91.85 & 101.5 & 1.6 & {$[-1.8,4.7]$} & {$[90.2,96.2]$} \\
\hline 100.94 & 99.3 & 1.8 & {$[-4.9,3.4]$} & {$[96.0,104.4]$} \\
\hline 111.03 & 100.4 & 1.9 & {$[-4.0,4.8]$} & {$[106.5,116.3]$} \\
\hline \multicolumn{5}{|c|}{ Sodium ascorbate ${ }^{\mathrm{a}}$} \\
\hline 71.01 & 100.4 & 1.8 & {$[-3.7,4.6]$} & {$[68.3,74.3]$} \\
\hline 79.01 & 98.9 & 1.7 & {$[-4.1,3.8]$} & {$[75.8,82.0]$} \\
\hline 84.50 & 99.5 & 2.1 & {$[-5.2,4.1]$} & {$[80.1,87.9]$} \\
\hline
\end{tabular}

\footnotetext{
a Expressed on ascorbic acid equivalence basis.
} 
I. Tomuţă et al: Quantification of ascorbic acid and sodium ascorbate in powder blends for tableting and in vitamin C chewable tablets by NIR-chemometry, Acta Pharm. 63 (2013) 373-384.

content and $4.6 \%$ for the lowest sodium ascorbate content of tablets in validation batches.

Figs. 2c and d represent linear profiles of the prediction models. Again, the linearity profile is within the acceptance limits, the $R^{2}$ value is higher than 0.95 and the slope is very close to 1 . This confirming the linearity of the models for ascorbic acid and sodium ascorbate assays in tablets.

\section{CONCLUSIONS}

According to the data presented, the NIR-chemometric methods using the (j) model for ascorbic acid and the (i) model for sodium ascorbate have good reproducibility and satisfactory accuracy and linearity profiles. After analyzing the statistical parameters, it can be concluded that both NIR-chemometric methods are linear and sufficiently precise and accurate for simultaneous and direct quantification of the two components (ascorbic acid and sodium ascorbate) in powder blends for tableting, and in chewable tablets.

In conclusion, the overall results of this paper indicate that near infrared spectroscopy methods are appropriate and advantageous for directly predicting, without any sample preparation, of ascorbic acid and sodium ascorbate in powder blends for tableting and in vitamin C chewable tablets. Such rapid NIR-chemometric methods can be used for on-line, in-line or at-line monitoring of the manufacturing process of vitamin $\mathrm{C}$ chewable tablets and are helpful in achieving the goals of the process analytical technology concept.

Acknowledgements. - This work was supported by the Iuliu Hatieganu University of Medicine and Pharmacy Cluj-Napoca, Romania, project number 27020/4/11.2011.

\section{REFERENCES}

1. C. Bodson, E. Rozet, E. Ziemons, B. Evrard, P. Hubert and L. Delattre, Validation of manufacturing process of Diltiazem $\mathrm{HCl}$ tablets by NIR spectrophotometry (NIRS), J. Pharm. Biomed. Anal. 45 (2007) 356-361; DOI: 10.1016/j.jpba.2007.05.015.

2. M. Blanco and A. Peguerfo, Influence of physical factors on the accuracy of calibration models for NIR spectroscopy, J. Pharm. Biomed. Anal. 52 (2010) 59-65; DOI: 10.1016/j.jpba.2009.12.009.

3. D. Christensen, M. Allesø, I. Rosenkrands, J. Rantanen, C. Foged, E. M. Agger, P. Andersen and H. M. Nielsen, NIR transmission spectroscopy for rapid determination of lipid and lyoprotector content in liposomal vaccine adjuvant system CAF01, Eur. J. Pharm. Biopharm. 70 (2008) 914-920; DOI: $10.1016 /$ j.ejpb.2008.07.007.

4. M. C. Sarraguça and J. A. Lopes, Quality control of pharmaceuticals with NIR: From lab to process line, Vib. Spectrosc. 49 (2009) 204-210; DOI: 10.1016/j.vibspec.2008.07.013.

5. A. S. ElHagrasy, S. Y. Chang and S. Kiang, Evaluation of risk and benefit in the implementation of near-infrared spectroscopy for monitoring of lubricant mixing, Pharm. Dev. Technol. 11 (2006) 303-312; DOI: 10.1080/10837450600767664.

6. I. Tomuta, R. Iovanov, A. L. Vonica and S. E. Leucuta, High-throughput NIR-chemometric method for meloxicam assay from powder blends for tableting, Sci. Pharm. 79 (2011) 885-898; DOI: 10.3797/scipharm.1108-07. 
I. Tomuţă et al.: Quantification of ascorbic acid and sodium ascorbate in powder blends for tableting and in vitamin C chewable tablets by NIR-chemometry, Acta Pharm. 63 (2013) 373-384.

7. C. V. Liew, A. D. Karande and P. W. Heng, In-line quantification of drug and excipients in cohesive powder blends by near infrared spectroscopy, Int. J. Pharm. 386 (2010) 138-148; DOI: 10. 1016/j.ijpharm.2009.11.011.

8. I. Tomuta, R. Iovanov, E. Bodokiand and S. E. Leucuta, Quantification of meloxicam and excipients on intact tablets by near infrared spectrometry and chemometry, Farmacia 58 (2010) 559_ 571.

9. A. Porfire, I. Tomuta, L. Tefas, S. E. Leucuta and M. Achim, Simultaneous quantification of 1- $\beta$ -phosphatidylcholine and cholesterol in liposomes using near infrared spectrometry and chemometry, J. Pharm. Biomed. Anal. 63 (2012) 87-94; DOI: 10.1016/j.jpba.2012.01.017.

10. L. Nováková, D. Solichová and P. Solich, Hydrophilic interaction liquid chromatography charged aerosol detection as a straightforward solution for simultaneous analysis of ascorbic acid and dehydroascorbic acid, J. Chromatogr. A. 1216 (2009) 4574-4581; DOI: 10.1016/j.chroma. 2009.03.060.

11. M. G. Gioia, P. Andreatta, S. Boschetti and R. Gatti, Development and validation of a liquid chromatographic method for the determination of ascorbic acid, dehydroascorbic acid and acetaminophen in pharmaceuticals, J. Pharm. Biomed. Anal. 48 (2008) 331-339; DOI: 10.1016/j.jpba. 2008.01.026.

12. United States Pharmacopeia 32, National Formulary 27, USPConvention, Rockville (MD) 2009.

13. European Pharmacopeia $7^{\text {th }}$ ed., EDQM, Strasbourg 2011.

14. J. Mantanus, E. Ziémons, P. Lebrun, E. Rozet, R. Klinkenberg, B. Streel, B. Evrard and P. Hubert, Active content determination of non-coated pharmaceutical pellets by near infrared spectroscopy: Method development, validation and reliability evaluation, Talanta 80 (2010) 1750-1757; DOI: $10.1016 /$ j.talanta.2009.10.019.

15. C. P. Meza, M. A. Santos and R. J. Romañach, Quantitation of drug content in a low dosage formulation by transmission near infrared spectroscopy, AAPS PharmSciTech 7 (2006) E206-E214; DOI: $10.1208 /$ pt070129.

16. P. Hubert, J. J. Nguyen-Huu, B. Boulanger, E. Chapuzet, P. Chiap, N. Cohen, P. A. Compagnon, W. Dewé, M. Feinberg, M. Lallier, M. Laurentie, N. Mercier, G. Muzard, C. Nivet and L. Valat, Harmonization of strategies for the validation of quantitative analytical procedures: A SFSTP proposal - Part I, J. Pharm. Biomed. Anal. 36 (2004) 579-586; DOI: 10.1016/j.jpba.2004.07.027.

17. P. Hubert, J. J. Nguyen-Huu, B. Boulanger, E. Chapuzet, N. Cohen, P. A. Compagnon, W. Dewé, M. Feinberg, M. Laurentie, N. Mercier, G. Muzard, L. Valat and E. Rozet, Harmonization of strategies for the validation of quantitative analytical procedures: A SFSTP proposal: Part IV. Examples of application, J. Pharm. Biomed. Anal. 48 (2008) 760-771; DOI: 10.1016/j.jpba.2008.07.018.

18. E. Ziémons, J. Mantanus, P. Lebrun, E. Rozet, B. Evrard and P. Hubert, Acetaminophen determination in low-dose pharmaceutical syrup by NIR spectroscopy, J. Pharm. Biomed. Anal. 53 (2010) 510-516; DOI: 10.1016/j.jpba.2010.06.003.

19. C. De Bleye, P. F. Chavez, J. Mantanus, R. Marini, P. Hubert, E. Rozet and E. Ziemons, Critical review of near-infrared spectroscopic methods validations in pharmaceutical applications, J. Pharm. Biomed. Anal. 69 (2012) 125-132; DOI: 10.1016/j.jpba.2012.02.003. 